\title{
Selection of Prime Actor in Humans during Bimanual Object Manipulation
}

\author{
Anna Theorin ${ }^{1}$ and Roland S. Johansson ${ }^{1,2}$ \\ ${ }^{1}$ Department of Integrative Medical Biology, Physiology Section and 2Umeå Center for Functional Brain Imaging, Umeå University, SE-901 87 Umeå, \\ Sweden
}

In bimanual object manipulation tasks, people flexibly assign one hand as a prime actor while the other assists. Little is known, however, about the neural mechanisms deciding the role assignment. We addressed this issue in a task in which participants moved a cursor to hit targets on a screen by applying precisely coupled symmetrical opposing linear and twist forces on a tool held freely between the hands. In trials presented in an unpredictable order, the action of either the left or the right hand was spatially congruent with the cursor movements, which automatically rendered the left or right hand the dominant actor, respectively. Functional magnetic resonance imaging indicated that the hand-selection process engaged prefrontal cortical areas belonging to an executive control network presumed critical for judgment and decision-making and to a salience network attributed to evaluation of utility of actions. Task initiation, which involved switching between task sets, had a superordinate role with reference to hand selection. Behavioral and brain imaging data indicated that participants initially expressed two competing action representations, matching either mapping rule, before selecting the appropriate one based on the consequences of the initial manual actions. We conclude that implicit processes engaging the prefrontal cortex reconcile selections among action representations that compete for the establishment of a dominant actor in bimanual object manipulation tasks. The representation selected is the one that optimizes performance by relying on the superior capacity of the brain to process spatial congruent, as opposed to noncongruent, mappings between manual actions and desired movement goals.

\section{Introduction}

Object manipulations commonly engage both hands in which one usually serves as a prime actor while the other has an assisting or postural function (Guiard, 1987; MacNeilage, 1987). The brain appoints a prime actor even in tasks that require the hands to apply coupled symmetrical opposing forces on a single object and, notably, the choice of prime actor is flexible and depends on the spatial mapping between hand forces and desired movement outcomes (Johansson et al., 2006). For example, when removing the lid from a jar, the hand that grasps the lid, be it left or right depending on overall task constraints, serves as prime actor because the twist force it generates is aligned with the goal motion, that is, the rotation of the lid. Likewise, when moving a cursor by symmetrically applying forces to a tool held freely between the hands to chase targets on a screen, after learning the task (Sailer et al., 2005), participants appoint either the left or the right hand as prime actor depending on whether the cursor moves directionally with left- or right-hand forces (Johansson et al., 2006). The appointment of a prime actor in a context-dependent manner apparently reflects a choice made by the brain in executing one of potentially available action representations that differentially

Received March 30, 2010; revised May 19, 2010; accepted June 14, 2010.

This work was supported by Swedish Research Council Project 08667 and 6th Framework Program of the European Union Project IST-028056. We thank Micael Andersson, Anders Bäckström, and Göran Westling for their technical support and Benoni Edin and Lars Nyberg for valuable comments on this manuscript.

Correspondence should be addressed to Roland S. Johansson, Physiology Section, Department of Integrative Medical Biology, University of Umeå, SE-901 87 Umeå, Sweden. E-mail: roland.s.johansson@physiol.umu.se.

DOI:10.1523/JNEUROSCI.1624-10.2010

Copyright $\odot 2010$ the authors $\quad$ 0270-6474/10/3010448-12\$15.00/0 transform goal motions represented in a visuospatial reference frame into hand motor commands. Change in hand assignment parallels a midline shift of lateralized activity in distal hand muscles, corticospinal pathways, and primary sensorimotor cortex (SMC), and related cerebellar areas (Johansson et al., 2006). Consistent with a central role of left-hemisphere processes for righthanders in performing skilled manual actions (Geschwind, 1975; Leiguarda and Marsden, 2000; Schluter et al., 2001), a leftlateralized dorsal parietal-premotor network provides critical neural substrates of the implemented action representation regardless of prime actor (Theorin and Johansson, 2007).

We asked whether hand selection in bimanual tasks is a function exclusive of the parietal-premotor networks in which stored action representations may compete against each other for behavioral expression until a single response is selected based on information accumulated that bias this competition (Schall and Thompson, 1999; Gold and Shadlen, 2001; Mazurek et al., 2003). Alternatively, both cortical and subcortical regions, including the prefrontal cortex and basal ganglia, may be engaged transitorily to reconcile this selection by facilitating the expression of a given action representation while suppressing competitors (Miller and Cohen, 2001; McCoy and Platt, 2005; Cisek, 2007). To address this issue, we collected functional magnetic resonance images representing the hand-selection phase in trials in which participants chased targets while the mapping between bimanual forces and cursor movements rendered either the left or right hand the prime actor. Because the prevailing mapping rule was initially unknown to the participants, they selected the appropriate action representation based on an evaluation of the cursor movements 

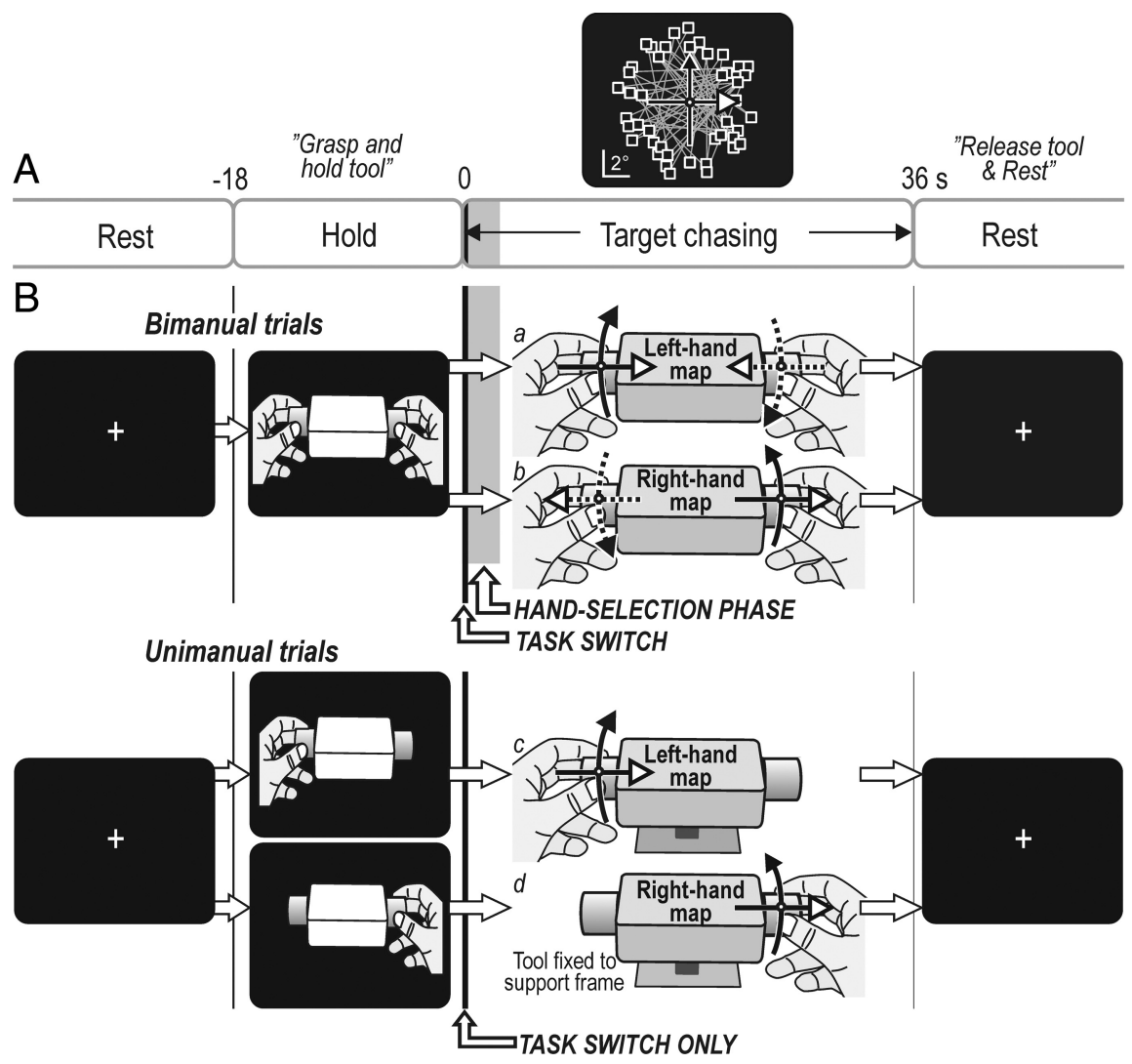

Figure 1. Apparatus, experimental design, and target distribution on screen. $\boldsymbol{A}$, Each trial involved a hold task (18 s) during which a picture instructed the participant how to grasp the tool (see $\boldsymbol{B})$, followed by the target-chasing task (36s) and a rest period (18s) in which the participant first released the tool and then rested while fixating a crosshair. The top graph shows the distribution of target positions (squares) with the cursor shown in the center of the screen (white dot). Straight lines connect consecutively appearing targets. The width of the vertical gray bar initially during the target-chasing task indicates the time of the hand-selection phase in bimanual trials (averaged across participants). The width of the vertical solid black line indicates the time between the appearance of the first target and the start of cursor movements (task initiation). $\boldsymbol{B}$, Tool used by the participants to control the cursor by bimanually ( $\boldsymbol{a}$ and $\boldsymbol{b}$ ) or unimanually ( $\boldsymbol{c}$ and $\boldsymbol{d}$ ) applying linear forces and torques to the tool. The corresponding solid line arrows in top graph of $\boldsymbol{A}$ indicate the two different mapping rules relating forces and torques to cursor movements, i.e., the left-hand and the right-hand mapping rule. For the unimanual trials ( $($ and $\boldsymbol{d} \boldsymbol{d}$ ), the left-hand and the right-hand mapping rule was applied when the left and right hand acted, respectively. Note that selection of the prime actor occurred initially during the target-chasing task only in the bimanual trials (hand-selection phase), whereas a switching from the hold task to the targetchasing task occurred for all trial types.

resulting from their own manual actions. We compared images in bimanual trials with matching images obtained when the same task was performed unimanually with either hand. Using a factorial design, we sought to detect areas with main effects of hand selection as well as interactions depending on whether the left or right hand was selected as the prime actor. In addition, using event-related images, we asked whether areas engaged in the hand selection differed from those that support task initiation, which involved a task switch from simply holding the tool to perform the actual target-chasing task.

\section{Materials and Methods}

Participants, apparatus, and general procedure

After providing written informed consent in accordance with the Declaration of Helsinki, eight males and eight females (age range, 23-37 years) participated. All were right-handed (Oldfield, 1971). A detailed description of the experimental procedure and the apparatus can be found in previous reports that address other aspects of data obtained in the same functional magnetic resonance imaging (fMRI) experiments (Johansson et al., 2006; Theorin and Johansson, 2007). In short, the target-chasing task involved moving a cursor (filled circle, diameter of $0.2^{\circ}$ visual angle) on a computer screen to hit, as fast as possible, succes- sively displayed targets (open square, side of $0.8^{\circ}$ ). A hit was registered when the cursor had been inside the target zone for $\geq 15 \mathrm{~ms}$. Once a target had been hit, it immediately appeared at a new unpredictable position on the screen (Fig. $1 A$, top). The distance between two successive targets was $8.8^{\circ}$, and the direction from the previous target was uniformly distributed between 0 and $360^{\circ}$.

To move the cursor, the participants applied forces either bimanually or unimanually to cylindrical handles (diameter of $3.4 \mathrm{~cm}$, length of $3 \mathrm{~cm}$ ) attached to each side of a rectangular box $(12 \times 8 \times 3.5 \mathrm{~cm})$ (Fig. $1 \mathrm{~B})$. The handles, gripped by the thumb, index, and long finger, were equipped with custom-built optometric force transducers that measured compressing and stretching forces along the longitudinal axis of the tool and twist forces around this axis (bandwidth: 0-120 Hz). Application of longitudinal and twist forces moved the cursor horizontally and vertically, respectively. Thus, combinations of longitudinal and twist forces were required to move the cursor diagonally on the screen. With zero force, the cursor was in the middle of the screen. On average, the targets were located $4.7 \pm 0.7^{\circ}$ (mean $\pm 1 \mathrm{SD}$ ) from the screen center, which corresponded to $2.2 \pm 0.3 \mathrm{~N}$ tangential force applied between the handles in the bimanual conditions or to the handle used in the unimanual conditions.

In the bimanual conditions (Fig. $1 B a, B b$ ), the tool was held unsupported between the hands, which implied that the two hands generated forces of virtually the same magnitudes but in opposite directions. Thus, forces generated by one hand were counterbalanced by opposing forces of corresponding magnitudes generated by the other hand. By tracking the position of reflex markers attached to the box of the tool, a CCD camera device measured the small lateral and rotational movements of the tool that occurred during the target chasing with a resolution better than $0.02 \mathrm{~mm}$ and $0.01^{\circ}$, respectively. In the unimanual conditions (Fig. $1 B c, B d$ ), the tool was fixated to a support frame. Importantly, an engaged hand generated nearly identical forces when pursuing targets whether acting bimanually or unimanually.

\section{Trials and experimental protocol}

Two different mapping rules linked hand forces and cursor movements. With one rule, "the left-hand rule," the cursor moved with the direction of the forces applied by the left hand (Fig. $1 B a, B c$ ). That is, it moved rightward when the participant applied longitudinal compression forces to the tool and leftward with stretching forces. Counterclockwise twist forces moved the cursor upward and clockwise twist forces downward. This mapping rule was applied in one of two bimanual conditions (Fig. $1 \mathrm{Ba}$ ) and when the participants used the tool unimanually with the left hand (Fig. $1 B c$ ). For the other mapping rule, "the right-hand rule," the cursor moved with the direction of the forces applied by the right hand (Fig. $1 B b, B d$ ). Thus, it moved leftward with longitudinal compression forces and upward with clockwise twist forces. This mapping rule was applied in one of the two bimanual conditions (Fig. $1 \mathrm{Bb}$ ) and when the participants used the tool unimanually with the right hand (Fig. $1 B d$ ). In the bimanual conditions, the participants automatically, i.e., without any instructions, soon after task commencement let their left hand be the prime actor with the left-hand rule and the right hand with the righthand rule (Johansson et al., 2006). 
Each trial included an $18 \mathrm{~s}$ period during which the screen displayed a drawing of the tool held by the left, right, or both hands (Fig. $1 \mathrm{~B}$ ). The hold task commenced when the drawing appeared and the participant's gripped the tool as indicated, lifted it $\sim 2 \mathrm{~cm}$ off its support and held it aloft while waiting for the target-chasing task to commence, triggered by the display instead showing the cursor and the first target (Fig. $1 \mathrm{~A}$ ). After $36 \mathrm{~s}$ of target chasing, the cursor and target extinguished. This signaled to the participant to replace and release the tool and rest while watching a crosshair that appeared in the center of the screen (Fig. 1B). After $18 \mathrm{~s}$ rest, a new trial started. Each scanning session (see below) began and ended with an $18 \mathrm{~s}$ rest period. Importantly, in the bimanual trials, the drawing shown during the hold task provided no information about which of the two mapping rules prevailing during the forthcoming target-chasing task and thus no information about which action representation to execute. In contrast, for the unimanual trials, the instruction about which hand to use was linked to the mapping rule and thus to the appropriate action representation. Hence, the appropriate action representation was specified before the commencement of the target-chasing run for the unimanual but not for the bimanual trials.

Before the scanning, the participants had practiced the four types of trials (Fig. $1 B a-B d$ ) for several days (Johansson et al., 2006). That is, they had learned each of the four action representations and could swiftly switch between them. During the scanning, each participant experienced four consecutive fMRI sessions that each included one trial of each type. The order of the four trial types in the sessions was unpredictable for the participants and counterbalanced across them. Between sessions, there were breaks of $2 \mathrm{~min}$ during which the participants were reminded verbally to hit the targets as fast as possible.

\section{fMRI parameters}

A whole-brain fMRI scan was collected every $3 \mathrm{~s}$ by using a $1.5 \mathrm{~T}$ Philips Gyroscan ACS NT scanner (Philips Medical Systems). Multislice $\mathrm{T} 2^{\star}$-weighted images (echo time, $50 \mathrm{~ms}$; flip angle, $90^{\circ}$; and in-plane resolution, $3.44 \times 3.44 \mathrm{~mm}^{2}$ in a $64 \times 64$ matrix) were provided by a gradient-echo sequence. An image volume comprised 33 continuous slices of $4.4 \mathrm{~mm}$ thickness with no interslice gap. To cope with T1 equilibration effects, before each scanning session, five surplus scans was collected while the screen displayed the crosshair.

\section{Data analysis}

Behavioral data. We measured the participants' performance during the target-chasing task by "hit time" and a cursor "path index" computed for each target directed movement. Hit time was the time it took to hit a target from the instance it appeared on the screen and thus included the "reaction time" and the time for the target-directed movement. The path index, which reflected the straightness of the cursor trajectory, was the ratio between the distance traveled by the cursor to the target and the straight-line distance to the target. To measure the evolution of the hand assignment during the bimanual target-chasing task, we first computed the time-varying correlation (moving time window of $\pm 1 \mathrm{~s}, 10 \mathrm{~ms}$ time steps) between longitudinal forces and lateral tool movements and between twist forces and tool rotations, respectively, as described previously (Johansson et al., 2006). We then used the mean of the obtained coefficients as a single time-varying measure of hand asymmetry. If the tool consistently moved with the forces applied by the left hand, this hand asymmetry index would attain a value approaching +1 , which indicated left-hand dominance during the target chasing. Conversely, values approaching -1 indicated that the tool consistently moved with the forces applied by the right hand, indicating right-hand dominance.

To assess the time when the cursor commenced moving toward a target, we used the second and third time differential of the forces applied to the tool. These time differentials were derived from the vector sum of the first time differentials of the longitudinal and twist forces after lowpass filtering at $16 \mathrm{~Hz}$. Empirically, we found that a reliable criterion for finding the time of the movement start was when the amplitude of the second time differential exceeded $50 \mathrm{~N} / \mathrm{s}^{2}$; the movement start was then defined as the closest positive peak of the third time differential.

For statistical analyses of the behavioral data, we used repeatedmeasures ANOVAs based on median values computed for each partici- pant as specified in Results. $p<0.05$ was considered statistically significant. Correlation coefficients were converted using Fisher's $z$ transformation. Post hoc analyses were performed with the Tukey's honestly significant difference test.

fMRI data. The blood oxygenation level-dependent (BOLD) signals provided by the scanner were analyzed with SPM5 (The Wellcome Department of Cognitive Neurology, London, UK; http://www.fil.ion. ucl.ac.uk/spm). The functional images were realigned and unwarped to correct for head movements, coregistered with each subject's anatomical MRI, and transformed (linear and nonlinear transformation) to the format of the Montréal Neurological Institute $(\mathrm{MNI})$ standard brain $(2 \times$ $2 \times 2 \mathrm{~mm}^{3}$ voxel size). The functional images were spatially smoothed with a $10 \mathrm{~mm}$ full-width at half-maximum isotropic Gaussian kernel, and a high-pass filtering $(2.3 \mathrm{mHz})$ reduced participant-specific signal drifts. We used proportional grand mean scaling over each scanning session to reduce the effect of any slow global changes in BOLD activity.

For each bimanual trial, we defined one "boxcar" regressor representing the hand-selection phase during which the participants selected the appropriate action representation rendering either the left or the right hand as the prime actor. This phase began at the start of the target chasing, and its duration, which could vary substantially between trials, was defined separately for each trial as described in Results. Corresponding regressors were defined for the unimanual trials in which the participants had selected acting hand before the target-chasing task commenced. That is, for each scanning session, the period of the hand-selection phase defined for the bimanual trial with the left-hand mapping rule was assigned to the unimanual trial when the left hand acted. Similarly, with the right hand acting unimanually, the regressor had the same duration as that of the hand-selection phase determined for the bimanual trial with the right-hand mapping rule. For each of the four trial types, we also defined one regressor representing the steady-state performance during the target-chasing task, i.e., the period that followed the regressor representing the hand-selection phase. Similarly, separate regressors modeled the different hold tasks (left-hand, right-hand, and bimanual grip). Results concerning the hold task and steady-state performance have been published previously (Theorin and Johansson, 2007). Therefore, these regressors represented states of no interest in the present study. After convolving the boxcar regressors with the standard canonical hemodynamic response function provided by SPM5, a general linear model was fitted to the data obtained for each participant ["first-level analysis" (Friston et al., 1999)]. We then entered the single-participant images into a random-effects group analysis ["second-level analysis" (Friston et al., 1999)]. In a $2 \times 2$ factorial design (ANOVA), we analyzed the main effects of the hand selection (bimanual vs unimanual grip) and mapping rule (left-hand, right-hand rule) and, importantly, interactions between these factors. The ANOVA approach allowed us to detect effects of interest in a single statistical test, whereas numerous tests would have been needed had we used the more traditional $t$ test approach. To resolve the nature of the main effects and interactions as indicated by the ANOVA, at the level of clusters, we examined BOLD effect sizes ( $\beta$ values and their sign) and time-varying changes of the BOLD signal expressed as percentage relative to mean of session.

To assess BOLD signal increases linked to task initiation, which implied a switching from a task set supporting the hold task to one supporting the target-chasing task, we applied an event-related analysis on the same fMRI data. For each trial, a stick function was aligned with the time the participant started to move the cursor to hit the first target. After convolving the stick function with the standard canonical hemodynamic response function, for each participant, we used the general linear model to obtain a functional image common for all four type of trials. Thus, the first-level analysis was based on 16 event-related images collected in each participant. Those images then went into a random-effects group analysis (second-level analysis). A one-sample $t$ test was used to label regions with increased BOLD activity. To check for possible effects of grip type (unimanual, bimanual) and of mapping rule (left-hand, right-hand rule) on the detected activations, for each subject, we measured the average BOLD signal change ( $\beta$ values) for the voxels of detected clusters and conducted repeated measures ANOVA on the BOLD activity. 
To protect against false positives while at the same time retain the power to detect statistically significant effects, we subjected the statistical images of the group analysis to a double-threshold approach, in which we combined a voxel-based threshold with a minimum cluster size (Forman et al., 1995). With our brain volume and an individual voxel threshold of $Z>2.88$, we used a cluster size corresponding to $p<0.05$ at the cluster level. This cluster threshold, corrected for multiple comparisons across the whole brain, was determined based on random field theory (Cao, 1999; Worsley et al., 1999, 2002) and implemented with the stat_threshold function of fmristat available at http://www.math.mcgill.ca/keith/ fmristat; the SPM software used did not allow cluster-based thresholding for ANOVA analyses. Our choice of voxel-based threshold reflected a tradeoff between underestimating the size of a focus of activation, identifying only the very peak of activations, versus joining distinct foci.

Based on coordinates provided in the MNI stereotaxic space, the anatomical localization of the detected clusters and their local maxima was initially assessed by the Automated Anatomical Labeling software (Tzourio-Mazoyer et al., 2002) and cross-referenced to the major sulci and gyri using the standard stereotactic atlas of Talairach and Tournoux (1988). We then validated this method of localization by superposing the $\operatorname{SPM}\{Z\}$ maps on the group mean anatomical MRI calculated after each individual's MRI had been stereotactically transformed into the same standard stereotactic space. When obvious differences were identified, we rectified the anatomical labeling of the detected effects. For areas of the mesial frontal lobe, we use the nomenclature proposed by Picard and Strick (2001) and for the lateral prefrontal cortex the nomenclature used by Ridderinkhof et al., (2004b). Local maxima had to be separated by at least $10 \mathrm{~mm}$. In-house developed software (DataZ; Physiology Section, Integrative Medical Biology, Umeå University, Umeå, Sweden) was used to visualize the effects.

\section{Results}

We present the results in three main sections. First, we analyze the participants' behavior in the scanner. We focus on the initial period of the target-chasing task while the selection and implementation of the prime actor took place and define the handselection phase as the period from the commencement of the target-chasing task until the appropriate action representation had been selected and implemented. We then examine regional BOLD signal changes linked to selection of the action representation that rendered either the left or the right hand as prime actor in the bimanual trials depending on the mapping between bimanual forces and cursor movements. To that end, we contrast images obtained during the hand-selection phase of the bimanual trials with the matching epoch during the unimanual trials in which the acting hand had been selected beforehand (Fig. 1). We based the analysis on a $2 \times 2$ factorial ANOVA because plausible experimental outcomes predicted possible interaction effects between grip type (bimanual vs unimanual grip) and mapping rule (left-hand vs right-hand rule). For example, the right-handed participants might have systematically appointed the right hand as the prime actor in the bimanual trials and consequently only had to change the action representation with the left-hand mapping rule. Finally, we examine activity related to initiation of the target-chasing task, which for all types of trials involved a switch from the task of holding the tool to the task of pursuing the sequentially displayed target on the screen.

\section{Behavior during the hand-selection phase}

To perform the target-chasing task, depending on grip type (bimanual, unimanual) and the mapping rule that related hand and cursor movements (left-hand or right-hand rule), participants implemented one of four previously learnt action representations (visuomotor transformations) (Fig. $1 B a-B d$ ). During the unimanual trials, the appropriate action representation was known before the task commenced because the mapping rule was linked to the acting hand, which grasped the tool during the hold task preceding the target-chasing task (Fig. $1 B c, B d$ ). In contrast, before the task commenced in the bimanual trials, the participants had no previous knowledge of which of the two mapping rules prevailed (Fig. $1 \mathrm{Ba}, \mathrm{Bb}$ ). We therefore predicted that the participants in the bimanual trials would initially produce defective target-directed cursor movements during the target-chasing task before the appropriate action representation could be selected guided by the sensory (visual) consequences of the produced manual actions. Indeed, especially when aiming for the first target in the bimanual trials, participants typically generated markedly erratic cursor movements (Fig. 2A, left), whereas the corresponding movements were rather straight in the unimanual trials (Fig. 2A, right).

For the movement to the first, second, and third targets, we quantified the adaptation to the prevailing mapping rule by analyzing the time it took to hit a target from the instance it appeared (hit time) and the straightness of the corresponding cursor trajectory (path index). In addition, to represent the steady-state performance during the target-chasing task, we computed the average hit time and path index for the target-directed movements for the second half of the task period. For the bimanual trials, both hit time and path index markedly and quickly declined with movement number (Fig. $2 B$, filled bars) but less so for the unimanual trials (Fig. $2 B$, open bars). A repeated-measures ANOVA with movement number (first, second, third, and steady state), grip type (bimanual, unimanual), and mapping rule (lefthand, right-hand) as fixed effects indicated an interaction between grip type and movement number (first, second, third, and steady state) on the hit time $\left(F_{(3,45)}=42.8 ; p<0.00001\right)$ and the path index $\left(F_{(3,45)}=55.0 ; p<0.00001\right)$. Post hoc analyses indicated a significant difference in both the hit time and the path index between the first movement in the bimanual trials and each of the subsequent movements ( $p<0.0002$ for all instances). Corresponding analyses for the unimanual trials failed to indicate a difference related to movement number. As reported previously, the steady-state performance was similar for all types of trials (Theorin and Johansson, 2007).

The initial improvement of the performance in the bimanual trials co-occurred with a development of functional hand dominance as assessed by the hand asymmetry index (Fig. 2C). Thus, movement number reliably affected the size of the hand asymmetry index $\left(F_{(3,45)}=126.5 ; p<0.00001\right)$, but the mapping rule did not. Post hoc analyses indicated that the hand asymmetry index differed between the first movement and the movements toward all subsequent targets ( $p<0.0002$ in all instances). In addition, the magnitude of this index for the movement to the second target was smaller than the index representing the steady-state performance $(p<0.01)$. The high hit time and path index as well as the low hand asymmetry index observed initially in the bimanual trials matched well the erratic target-directed movements executed before the proper action representation had been selected (Fig. 2A, left column). In contrast, when bimanual trials were performed in collateral experiments with the prevailing mapping rule known beforehand, the performance from the very beginning of the target-chasing run, including the hand asymmetry index, was practically identical to that observed during the end of the run.

It seems reasonable to assume that the selection of an appropriate action representation in the bimanual trials was based on information obtained about the current state of the apparatus by observing the cursor movements resulting from the forces ap- 
A
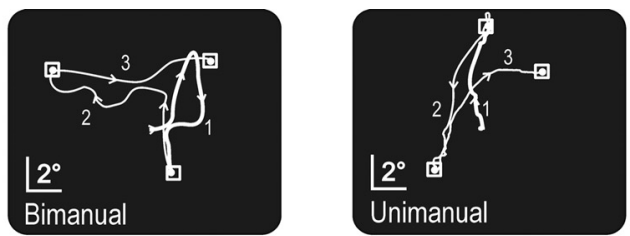

B

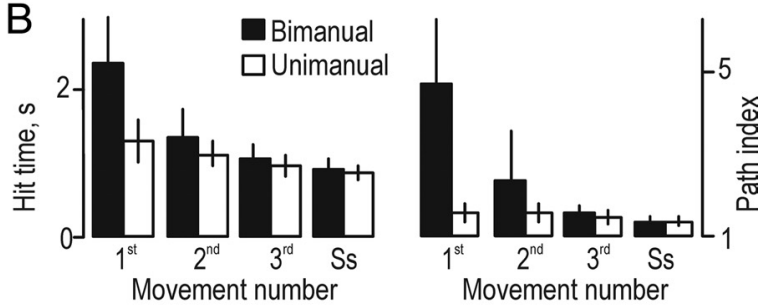

C
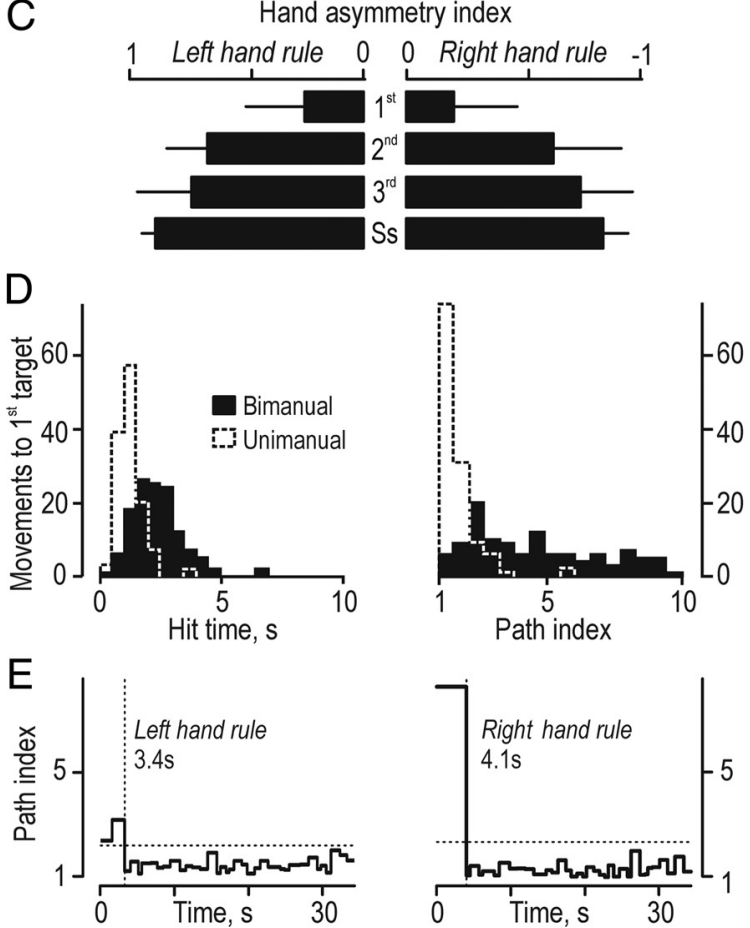

Figure 2. Participants' performance in the scanner. $\boldsymbol{A}$, Cursor trajectories exemplified for movements toward the first, second, and the third targets for a bimanual and a unimanual trial by a single participant. The thick segment of the movement trajectory represents the movement toward the first target (1). Arrowheads indicate the direction of cursor movement. $\boldsymbol{B}$, Hit time and path index for the first, second, and third movements and for movements to targets during the steady-state performance $(S s)$ recorded in bimanual and unimanual trials. Heights of columns give mean values computed across participants for data pooled across the left- and the right-hand mapping rule, and error bars indicate 1 SD $(n=16)$; mapping rule influenced neither hit time nor path index (data not shown). C, Hand asymmetry index recorded during the bimanual trials. Positive and negative values indicate by which degree left and right hand served as prime actor, respectively. Data compiled across participants as in B. D, Distribution of hit time and path index for the first movement for bimanual and unimanual trials by all participants ( $n=128$ ). $\boldsymbol{E}$, Assessment of the hand-selection phase during the bimanual trials exemplified by data from a single trial with each mapping rule by one participant. Dotted horizontal lines indicate the criterion value (3 SD above the mean during the steady-state period), and the dotted vertical line illustrates the time when the appropriate mapping rule was considered being implemented after the commencement of the target-chasing task (time 0 ). The numbers give the estimated duration of the hand-selection phase for each trial.

plied to the tool early during the target-chasing task. There are, however, other possible selection strategies. For instance, the participants could have appointed systematically either the left or the right hand as prime actor before the start of the target chasing, or they could have selected either hand based on guessing. With both these strategies, for one-half of the trials, the performance during the movement toward the first target would resemble that in the unimanual trials, whereas markedly inflated hit times and path indices would characterize the other half. However, the distribution of the hit time and the path index for the movements to the first target showed no obvious signs of such bimodality (Fig. $2 D)$. We therefore conclude that the participants selected the appropriate action representation in the bimanual trials based on information obtained after task initiation.

For most bimanual trials, the path index abruptly declined after the first target hit but occasionally after the second or the third hit (Fig. $2 E$; see also $B$, right). We assumed that this decline signified that the appropriate action representation had been selected and implemented. The period from the commencement of the target-chasing task until the time of this decline was termed the hand-selection phase. Inspection of single-trial data indicated that the duration of this phase could vary substantially between trials. To consider this variation in the analysis of the fMRI data (see further below), for each trial we estimated the duration of the hand-selection phase. Operationally, we defined its end as the instance the path index went below a criterion value computed as the mean plus 3 SDs of the path indices for all target transitions during steady-state performance (Fig. 2E, dashed lines). Although the hit time showed a similar decline as the path index, we used the path index because the decline in hit time was less conspicuous and the hit time for the first target was influenced by a prolonged reaction time related to task switching (see further below).

The duration of the hand-selection phase was $3.0 \pm 0.9 \mathrm{~s}$ (mean \pm SD and range of 1.3-4.5 s of median values computed for each participant, data pooled across mapping rules; $n=16$ ). In two-thirds of the bimanual trials (67\%), hand selection took place during the movement toward the first target. For most remaining trials, it occurred during the movement toward the second target, and, on average, it had taken place after $1.5 \pm 0.8$ target hits (mean \pm SD for data pooled across all bimanual trials by all participants, $n=128$ ). The duration of the hand-selection varied markedly between trials within participants. On average, this variation corresponded to an SD of $1.9 \mathrm{~s}$ (range of $0.6-2.7 \mathrm{~s}$ across participants; $n=16$ ). Mapping rule did not affect the duration of the hand-selection phase in the bimanual trials $\left(F_{(1,15)}=1.82 ; p=0.20\right)$.

When asked after the experiments, participants indicated that they had little impression that one hand might have played a dominant role during the bimanual trials and certainly not that the right hand was leading in some trials and the left hand in other. Thus, an implicit process essentially governed the hand selection.

\section{BOLD activity linked to the hand-selection phase}

To identify brain areas involved in hand selection, we analyzed BOLD signals recorded from the whole brain during the handselection phase in the bimanual trials and during the corresponding epochs in the matching unimanual trials, for which the acting hand was appointed beforehand (Fig. 1B) (see Materials and Methods). The factorial ANOVA used for the statistical analysis indicated a main effect of grip type bilaterally in prefrontal, primary sensorimotor, and posterior parietal cortical areas (supplemental Table S1, available at www.jneurosci.org as supplemental material). These areas were all more activated in the bimanual than in the unimanual trials, suggesting a role in the handselection process. A main effect of mapping rule was restricted to SMC and functionally related cerebellar regions (supplemental 
A
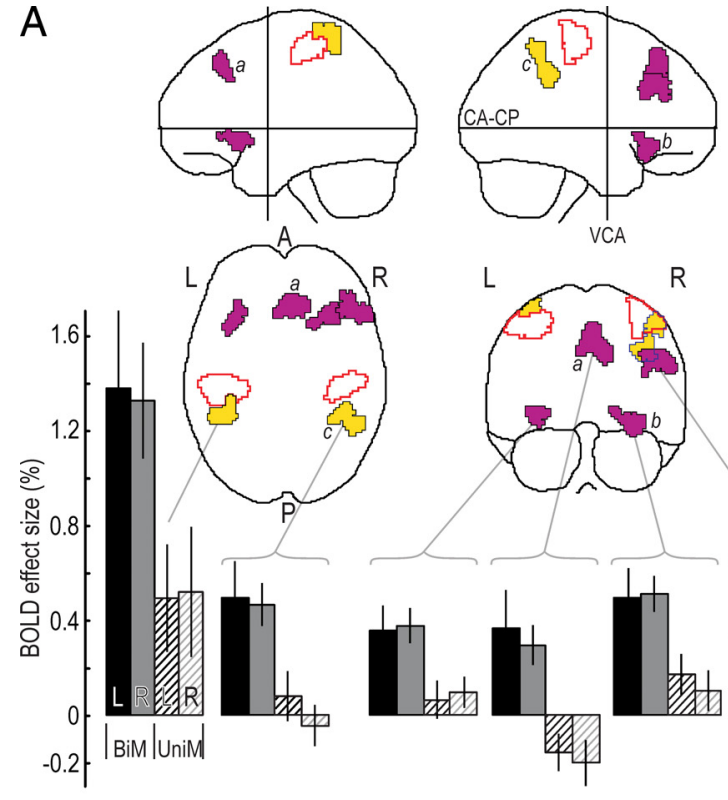

VCA

$\mathrm{B}$
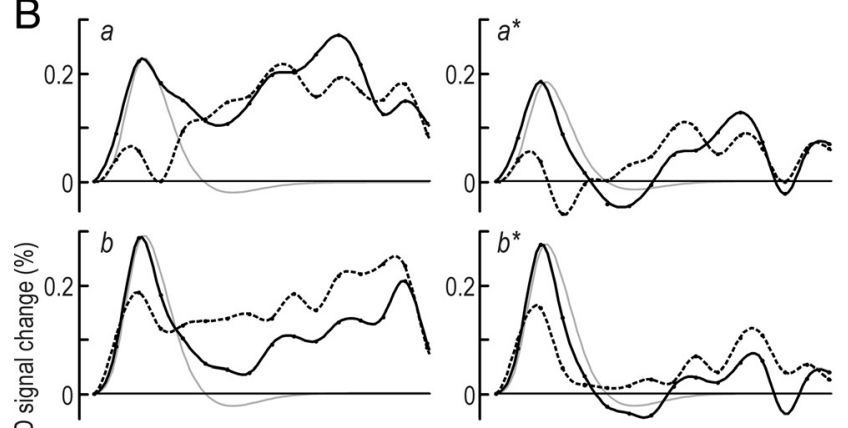

ㅁำ
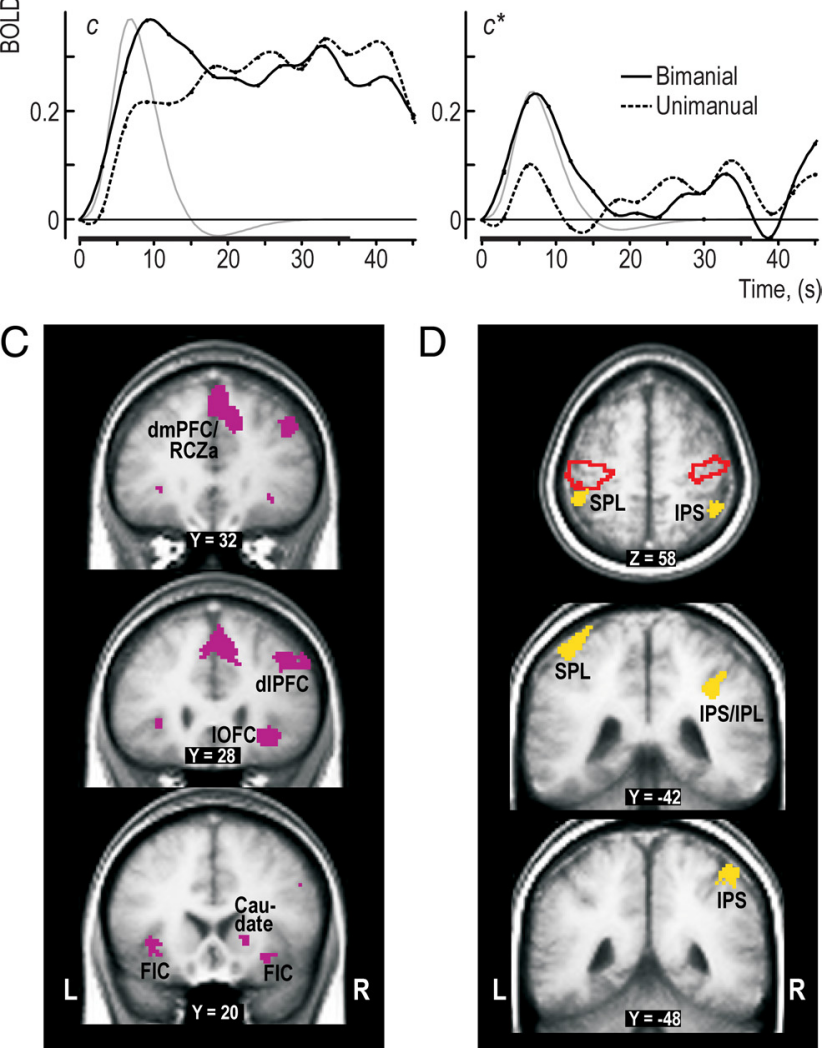

Figure 3. Prefrontal and posterior parietal areas engaged during the hand-selection phase in the bimanual trials. $\boldsymbol{A}$, Clusters with a main effect of grip type (bimanual, unimanual) superimposed
Table S2, available at www.jneurosci.org as supplemental material). Important interactions between grip type and mapping rule on the BOLD signal occurred in these areas, whereas no interactions were detected in the prefrontal and posterior parietal areas (supplemental Table S3, available at www.jneurosci.org as supplemental material).

\section{Hand selection engaged prefrontal and posterior parietal brain areas}

Four areas in prefrontal cortex showed stronger activation during bimanual compared with unimanual trials during the handselection phase (Fig. $3 A, C$, purple). One (275 voxels) involved the right dorsolateral prefrontal cortex (dlPFC), engaging the middle frontal gyrus with extensions into the pars triangularis of the inferior frontal gyrus and the inferior frontal sulcus. Another area (372 voxels) engaged bilaterally the dorsomedial prefrontal cortex (dmPFC), as well as the underlying anterior rostral cingulate zone (RCZa). For the ventral prefrontal cortex, we observed bilateral activation (110 and 204 voxels in the left right and right left hemispheres, respectively) of primarily the lateral orbitofrontal cortex (lOFC) that extended into the anterior insular gyrus [jointly referred to as the fronto-insular cortex (FIC)]. A rim of the cluster observed in the right hemisphere also extended into the caudate nucleus. In all these prefrontal areas, the timevarying BOLD signal showed a more marked peak initially during the target-chasing runs in the bimanual trials than in the matching unimanual trials (Fig. $3 B a, B b$ ).

We also detected stronger BOLD activity during the handselection phase of the bimanual trials in the right inferior parietal lobule (IPL) (210 voxels) principally located anterior in the intraparietal sulcus (IPS) and anterior in the left posterior parietal cortex, primarily in the superior parietal lobule (Fig. $3 A, D$, yellow areas; see also $B c$ ). This parietal activation, especially in the right IPL, agrees with imaging evidence indicating a functional connection with the right dlPFC (Fincham et al., 2002; Dosenbach et al., 2006). The left posterior parietal activation constituted a posterior part of a dumbbell cluster (830 voxels) extending into the SMC. For purposes of analysis, we conveniently split this cluster into an anterior and posterior section by applying a slightly more conservative voxel-based threshold $(Z>3.09$; see Materials and Methods). The pattern of BOLD signal changes in the part belonging to the posterior parietal cortex was similar to that ob-

\footnotetext{
on the MNI "glass brain." Purple and yellow areas indicate clusters located in prefrontal and posterior parietal cortical areas, respectively, and areas outlined in red depict mark cluster in the SMC regions. Histograms show, for each grip type (BiM, bimanual; UniM, unimanual) and mapping rule (L, left-hand; $R$, right-hand), the $B O L D$ effect sizes ( $\beta$ values) in percentage relative to mean BOLD signal level during the session. Height of columns gives mean value across participants, and vertical lines represent 1 SE $(n=16)$. $B$, Left column shows the time course of the BOLD signal averaged across all voxels in the clusters labeled by $\boldsymbol{a}, \boldsymbol{b}$, and $\boldsymbol{c i n} \boldsymbol{A}$ for data obtained in the bimanual and unimanual trials, respectively. The signal change is given in percentage relative to mean of session, and the curves are aligned to zero at the onset of the target-chasing task. The thin gray curve shows the time course of the hemodynamic response function corresponding to the regressor representing the hand-selection phase averaged across participants and arbitrarily scaled. Right column shows the corresponding BOLD data after the variation in the BOLD signal explained by the other regressors in the model was factored out, thus providing a view of what the regressor representing the hand-selection phase can capture. The embossed segment of the abscissa indicates the period of the target-chasing task. Black dots indicate interscan intervals. C, D, Main effect of grip type within the prefrontal cortex (purple) and posterior parietal cortex (yellow) shown on coronal and transversal slices of the averaged brain calculated across the participant-specific T1-weighted images $(n=16)$ after being normalized to the MNI brain template. SPL, Superior parietal lobule; IPS, intraparietal sulcus; $R$, right; L, left; $A$, anterior; $P$, posterior.
} 
served for the right anterior intraparietal sulcus (compare with Fig. $3 B c$ ), but the overall BOLD effect sizes were larger (Fig. $3 A$, bottom left).

An inspection of the time-varying BOLD signals in the prefrontal and parietal areas apparently involved in the hand selection suggested that they also showed increased activity during the entire target-chasing task (Fig. 3B). However, for the prefrontal areas, this sustained activity was not robust enough across participants to be significant in a random-effects group analysis we performed previously for the period of steady-state performance (Theorin and Johansson, 2007). In the face of such tendencies to sustained activity, we conservatively assessed BOLD signal changes during the hand-selection phase by running an ANOVA corresponding to our main ANOVA but using images that had been contrasted in the first-level analysis with images representing the steady-state performance. Importantly, this analysis revealed virtually identical activations as those illustrated in Figure 3 for prefrontal and parietal brain regions (data not shown).

Signals in primary sensorimotor cortex suggest simultaneous implementation of competing action representations

In addition to a main effect of grip type bilaterally in the SMC (Figs. $3 A, C, 4 A$, zones outlined with red), grip type and mapping rule significantly interacted on the BOLD signals in these areas during the hand-selection phase (blue areas in Fig. 4A). The BOLD effect size was similar regardless of mapping rules in the bimanual trials and in the unimanual trials when the contralateral hand acted, whereas it was much weaker when the ipsilateral hand acted in the unimanual trials (see BOLD effect sizes in Fig. $4 A$, left). Notably, for the right hemisphere, the zone of interaction, extending from the postcentral sulcus across the central sulcus and well into dorsal premotor areas, was much larger (2058 voxels) than that in the left hemisphere ( 248 voxels), which primarily engaged the central sulcus (Fig. $4 A$, blue areas). The difference in the BOLD signals during the unimanual actions also accounted for a main effect of mapping rule bilaterally in the SMC (Fig. 4A, yellow zones). That is, there was an overall stronger activation in the left hemisphere with the right-hand rule compared with the left hand-rule and, conversely, a stronger activation in the right hemisphere with the left-hand rule.

Inspection of the time-varying BOLD signals verified a similar activation in the SMCs during the hand-selection phase of the bimanual trials with both mapping rules (Fig. $4 B$, solid curves). However, after hand selection, the activity in the SMC ipsilateral to the prime actor decreased, whereas it was maintained in the contralateral SMC. As shown previously, the SMC activation during steady-state performance is significantly stronger when the contralateral hand is appointed as prime actor than when it plays an assisting rule (Johansson et al., 2006).

That SMC was bilaterally activated to a similar degree early in the bimanual trials regardless of mapping rule suggest that the action representations yielding right-hand and left-hand dominance were simultaneously executed before the appropriate representation was selected. Activation of both action representations is also consistent with the irregular and erratic cursor movements observed during the hand-selection phase (Fig. $2 A, B)$. Furthermore, a conjunction based on images collected for all four type of trials revealed that virtually the same parietalpremotor networks were activated during the hand-selection phase (Fig. 5A) as that supporting steady-state target chasing regardless of primarily acting hand (Theorin and Johansson, 2007). That is, dorsal premotor and posterior parietal areas were activated under all conditions and most extensively within the left
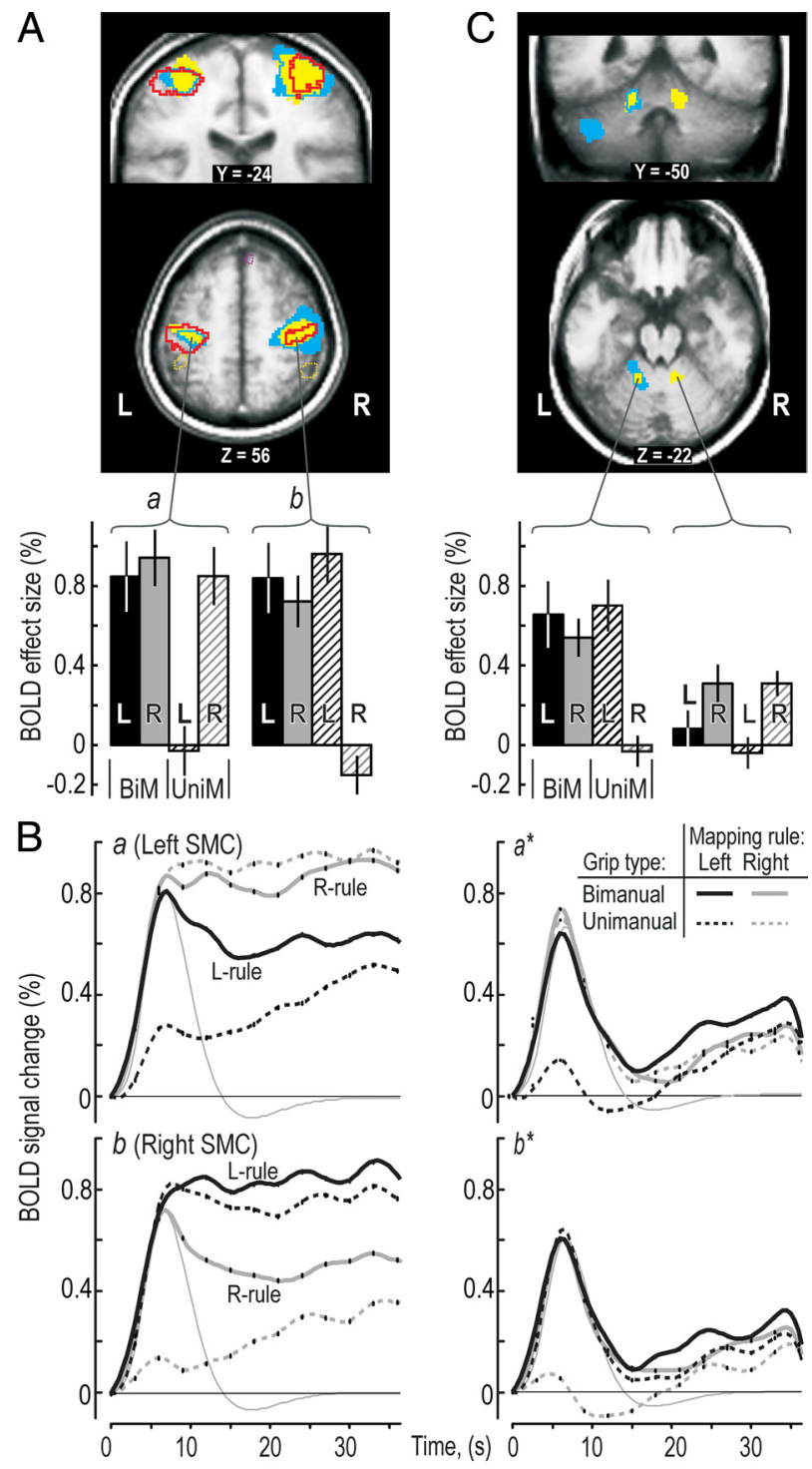

Figure 4. BOLD signals in primary sensorimotor areas (SMC) during the hand-selection phase. $A$, Regions with a main effect of grip type (red outline), mapping rule (yellow), and interactions between these experimental factors (blue areas) shown on coronal and transversal slices through the SMC obtained as in Figure 3C. Purple and yellow dashed lines indicate clusters with a main effect of grip type in prefrontal and posterior parietal areas, respectively. Histograms below show, for each type of trial, BOLD effect sizes in the clusters with the main effect of grip type (BiM, bimanual; UniM, unimanual; $L$, left; $R$, right) obtained and presented as in Figure $3 A$. B , Time course of the BOLD signals during the performance of the target-chasing task averaged across all voxels in the clusters labeled by $\boldsymbol{a}$ and $\boldsymbol{b}$ in $\boldsymbol{A}$ for data obtained for each type of trial. Format and data processing as in Figure $3 B$. C, Effect of mapping rule (yellow areas) and significant interaction between grip type and mapping rule (blue areas and outlines) observed in cerebellum. Identified clusters shown on coronal and transversal slices. BOLD effect sizes as in the bottom of $\boldsymbol{A}$.

hemisphere. Importantly, the premotor activity not only involved dorsolateral premotor cortex but also the supplementary motor areas (SMA proper and pre-SMA), the caudal cingulate zone $(\mathrm{CCZ})$, and the posterior rostral cingulate zone (RCZp) of the mesial frontal cortex (Fig. $5 B$ ). We also detected activity bilaterally in the occipital lobe and at the occipitotemporal junctions corresponding to the localization of the human motion complex (visual cortical area 5) implicated in perception of moving stimuli. For subcortical areas, we observed bilateral activity in thalamus and putamen (data not shown). 


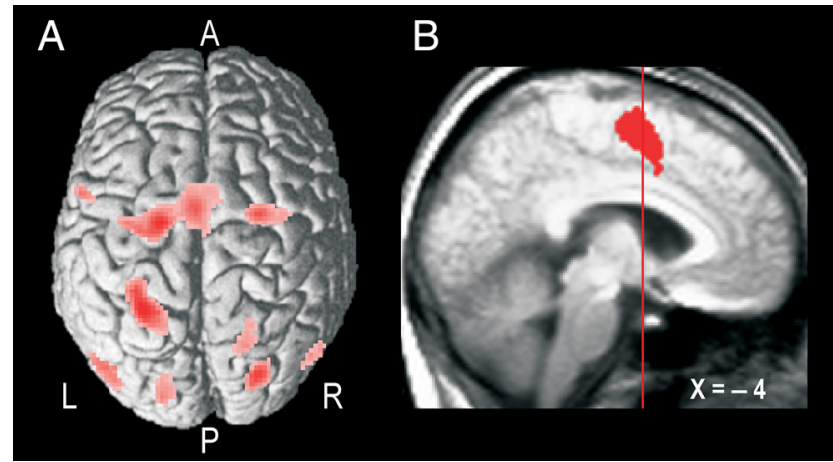

Figure 5. Parietal-premotor cortical areas engaged during the hand-selection phase. $A$, Common activations in all trial types averaged across participants rendered on a singlesubject standardized brain template in SPM2 viewed from a dorsal aspect (render depth, $20 \mathrm{~mm}$; L, left; R, right; A, anterior; $P$, posterior). $\boldsymbol{B}$, Common mesial frontal cortex activation shown on a sagittal slice of the brain obtained as described in the legend of Figure $3 C$. The vertical line indicates the position of the vertical plane passing through the anterior commissure. The depicted cluster involved both the superior frontal gyrus (BA 6; local maximum at $-4,-4,58$ ) and the middle cingulate gyrus (BA $24 ;-4,6,36$ )
Mapping rule affected the BOLD signals in the anterior "hand areas" of the intermediate cerebellum during the hand-selection phase (Fig. 4C, right, yellow areas). For the left cerebellum, the pattern of activation resembled that observed for the right primary SMC, including a significant interaction between grip type and mapping rule (Fig. 4C, blue areas intersecting yellow areas). However, the corresponding hand area of the right cerebellum showed no such interaction. Conversely, other patterns of interactions between grip type and mapping rule were observed bilaterally in more lateral cerebellar areas (Fig. 4C, top) and in the left brainstem (supplemental Table S3, available at www.jneurosci. org as supplemental material). Overall, these interactions involved stronger BOLD activity in bimanual trials with the righthand mapping rule and in unimanual trials with the left hand than in the other two trial types.

\section{Task set switching and related BOLD activity}

So far, we have addressed BOLD effects that correlated with the selection of action representations in the bimanual trials rendering either the left or right hand the dominant actor in the targetchasing task. However, before this selection could take place, participants first had to initiate the target-chasing task. That is, triggered by the shift in the display, the information processing of the brain changed from supporting executing the hold task to supporting the target chasing (Fig. 1). Thus, the brain switched task set, which involved both a change in the representation of the task goal, from holding the tool while waiting for the first target to appear on the screen and to chase targets, and a change in the parameters, or constraints, that implemented the lower-level sensorimotor processes that supported the ongoing target chasing. A reaction time analysis indicated that the neural processes responsible for the initiation of the movement to the first target that included task switching were more computationally demanding than the processes responsible for initiation of the targetdirected movements during ongoing target chasing (Fig. 6A). Averaged across all four types of trials, the time between the appearance of the first target on the screen and the start of the target-directed cursor movement was $0.44 \pm 0.11 \mathrm{~s}$ (mean \pm 1 SD of data from each participant, $n=16$ ), which was approximately twice the time for initiating a cursor movement to the subsequently appearing targets $(0.25 \pm$ $0.03 \mathrm{~s}$ ). A three-factorial repeated-measures ANOVA showed a main effect of the movement number (movement toward the first, second, and third targets and movements in the steady-state phase, $\left.F_{(3,45)}=47.6 ; p<0.00001\right)$ on the movement initiation time but failed to show an effect of grip type (bimanual, unimanual) and mapping rule (left-hand, right-hand), and there were no interaction effects. The initiation time for the movement to the first target was longer than that for the subse-
Figure 6. Brain regions involved in task switching. $\boldsymbol{A}$, Time for initiating cursor movements to a target appearing at an unpregive mean values computed across participants for data pooled across the left-hand and right-hand mapping rule, and error ba indicate 1SD ( $n=16)$; mapping rule influenced neither hit time nor path index (data not shown). $\boldsymbol{B}$, The MNI glass brain shows brain areas activated during initiation of the target-chasing task (orange areas) and, for comparison, an outline of prefrontal areas activated during the hand-selection phase in the bimanual trials (purple contours). $\boldsymbol{C}$, The same brain regions as in $\boldsymbol{A}$ shown on coronal, sagittal, and transversal slices of the brain obtained as described in the legend of Figure $3 C$. Vertical line on sagittal slices indicates the position of the vertical plane passing through the anterior commissure. Put., Putamen; P0J parieto-occipital junction. $\boldsymbol{D}$, Time course of the BOLD signals during performance of the target-chasing task averaged across all voxels in the clusters labeled by $\boldsymbol{a}, \boldsymbol{b}$, and $\boldsymbol{c}$ in $\boldsymbol{B}$ for data obtained during each type of trial. Format and data processing as in Figure $3 B$.

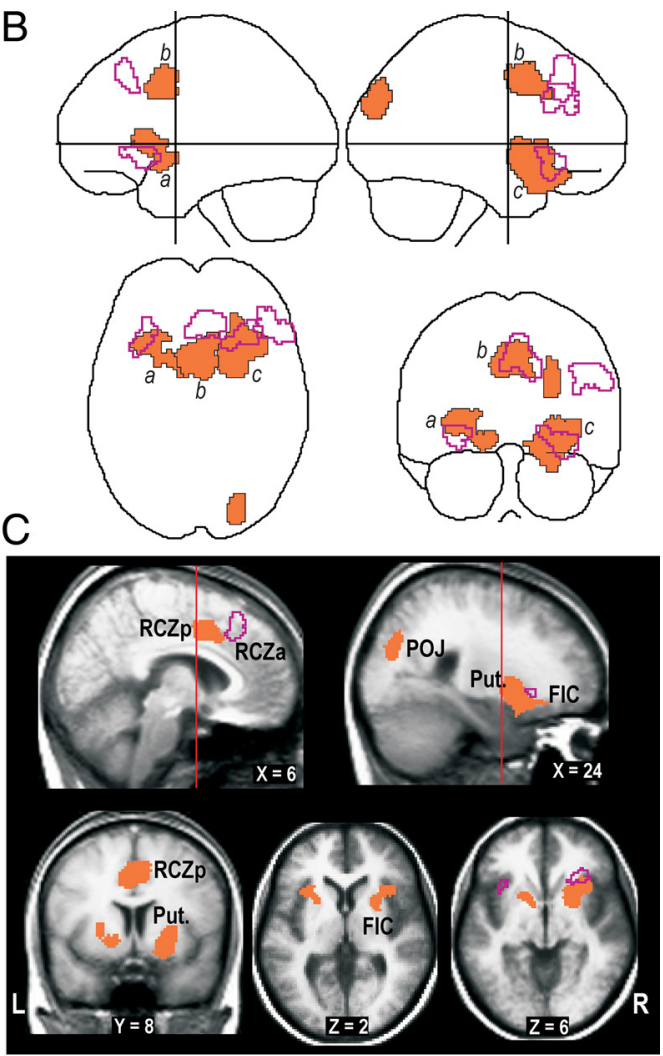


quent targets ( $p<0.0002$ in all cases), for which post hoc analyses showed no difference (Fig. 6A).

To identify brain areas engaged in switching between task sets, we looked for regional BOLD signal increases using event-related images synchronized with the initiation of the cursor movements directed to the first target. Because neither mapping rule nor grip type influenced the time required to switch between task sets, we assumed that similar brain processes were involved regardless of trial type and pooled data across the four types of trials in this analysis (see Materials and Methods). The ANOVA we used to detect BOLD signal changes related to hand selection would not detect signals associated with the task switching because such switches would occur in all trial types. Neither would such switches straightforwardly appear in the conjunction of images representing the hand-selection phase because of the substantial jitter in its duration and prolonged time (see above).

We detected four distinct brain areas associated with the task switching, three of which involved the prefrontal cortex and one involving the right superior occipital gyrus and the parietooccipital junction (Fig. $6 B, C$ ) (for details, see supplemental Table S4, available at www.jneurosci.org as supplemental material). One frontal activation (681 voxels) engaged bilaterally the RCZp, adjoining the pre-SMA, whereas two signified bilateral involvement of the anterior insula, ventral striatopalludum and putamen. The larger of the fronto-insular clusters (1134 voxels) (Fig. $6 \mathrm{Bc}$ ) was located in the right hemisphere and extended into IOFC. These frontal activations were overall located posterior to the prefrontal activations associated with hand selection in the bimanual trials (Fig. $6 B, C$, purple contours). Thus, for the mesial wall activation, task switching engaged the RCZp/pre-SMA rather than the $\mathrm{RCZa} / \mathrm{dmPFC}$ (Fig. 6C, top left), corroborating the notion that "selection for action" involves the RCZp, whereas $\mathrm{RCZa}$ is rather involved in evaluative functions (Picard and Strick, 2001). In fact, we only observed overlap with the frontal areas related to hand selection for a small segment ( 84 voxels) of the right FIC (Fig. 6C, bottom right). The more posterior localization of the areas associated with task switching compared with hand selection suggests different underlying networks. Furthermore, that grip type (bimanual vs unimanual) did not influence the time required to switch between task sets (Fig. 6A) suggests that the processes supporting hand selection were subordinate to those mediating switch of task set. Indeed, a three-way repeatedmeasures ANOVA run on the BOLD signal changes ( $\beta$ values) observed in the four identified clusters (factored in as four levels) failed to indicate a main effect of grip type $(p=0.19)$. Neither was there a main effect of mapping rule $(p=0.87)$, nor any twoor three-way interactions.

Inspection of the time-varying BOLD signals revealed that all areas identified as engaged in task switching showed a transient activation at the start of the target-chasing run regardless of trial type (Fig. 6D). However, in addition to the transient activation, these brain areas also tended to show sustained activity during the entire period of the target-chasing task (Fig. 6D), which agrees with our previous results that the pre-SMA/RCZp and the striatum also were activated during steady-state performance (Theorin and Johansson, 2007). Such sustained activity, which also applied to the areas related to hand selection (Fig. $3 B$ ), corroborates the general notion that prefrontal areas might exhibit "setmaintenance" activity so as to help upholding the control processes required for completing the task at hand (Miller and Cohen, 2001; Dosenbach et al., 2006). In the target-chasing task, this would have included upholding the visuomotor processes supporting the sequential intertarget movements both with re- gard to the adaption of the metrics of the underlying motor commands for the current target position (the target popped up at a new place of the screen after each hit) and to the initiation and execution of each movement.

\section{Discussion}

The central advance of this study is the demonstration that the implicit neural processes supporting role assignment of the hands in bimanual object manipulation can engage brain circuitry traditionally both associated with executive functions controlling and managing various conscious cognitive processes and the evaluation of utility of actions. Furthermore, these processes, functionally subordinate to those supporting switching between tasks, enhance performance by optimizing the use of the intrinsic capacities of the brain for performing sensorimotor transformations.

In the present study, participants performed the same targetchasing task under four different conditions. That is, one of four action representations, or sensorimotor transformations, was implemented depending on context. The context in the unimanual trials strongly favored a selection of an action representation before the commencement of the task, whereas in the bimanual trials, two representations were potentially affordable with an equal probability at task commencement. The bilateral activation of SMC and the erratic cursor movements indicate that the brain implemented both representations before singling out the desired one. Thus, the brain may not only prepare for a set of potential actions from which the actual one is selected before task execution (Cisek and Kalaska, 2005; Koch et al., 2006) but may also execute multiple action representations for the subsequent selection of the most desirable one. By activating both action representations, either would be more available for a rapid selection than had one been suspended beforehand. Furthermore, activating both representations presumably served an exploratory function, permitting early evaluation of the utility of either in the existing context.

We originally hypothesized that hand selection in the bimanual trials was a self-emergent property of the parietal-premotor system governing the task regardless of primary acting hand (see Introduction). Our results suggest, however, that prefrontal circuitry was engaged to reconcile this selection. That each of the optional action representations had to compete with a strong, equally probable, alternative probably favored recruitment of prefrontal processes (Miller and Cohen, 2001). Such processes can provide signals that influence and constrain computations occurring in the parietal-premotor pathways and thereby impose "decision factors" aiding goal-directed performance (Gold and Shadlen, 2001; McCoy and Platt, 2005; Cisek, 2007). Prefrontally mediated control of executive functions is typically implicated in situations in which participants are challenged with tasks in which externally imposed rules are learned to deliberately select in each trial one of alternative responses (Bunge, 2004; Ridderinkhof et al., 2004a; Rushworth et al., 2007). However, because there was no competition among response targets in the present targetchasing task, our results indicate that the brain also can engage prefrontal processes in implicit decisions supporting optimization of the use of its intrinsic sensorimotor processing capacities. That is, in the bimanual trials, the brain apparently engaged prefrontal circuitry to facilitate selection of a sensorimotor transformation that exploited the entrenched control systems of the brain for spatially congruent mappings between visual targets and matching motor commands for goal-directed actions. Controlling movements under noncongruent situations would have both 
increased the computational demands (Iacoboni et al., 1996; Jiang and Kanwisher, 2003) and resulted in an inferior performance (Fitts and Deininger, 1954; Kornblum et al., 1990; Dutta and Proctor, 1992; Wise and Murray, 2000).

The engagement of dlPFC, dmPFC, and functionally related posterior partial areas suggests that hand selection was supported by a canonical network termed the "executive control network" (Seeley et al., 2007; Sridharan et al., 2008; Habas et al., 2009). This network is considered critical for judgment and decision-making guided by pertinent sensory information as various action representations are weighed against shifting conditions and background homeostatic demands until specific actions are selected. Consistent with its activation pattern during hand-selection phase, dlPFC seems especially important in the presence of competing action representations (Rowe et al., 2000; Hadland et al., 2001; Schumacher et al., 2003). Furthermore, the engagement of primarily the right dlPFC and the functionally interconnected right IPL (Fincham et al., 2002; Dosenbach et al., 2006) corroborates that the right hemisphere has a prime role in action planning under conditions of spatial uncertainty (Schumacher et al., 2003; Weiss et al., 2006; Goel et al., 2007) and especially if exploratory processes and external cues are involved (Goldberg et al., 1994; Richards and Chiarello, 1997). In contrast, the left dlPFC would principally handle nonspatial selections (Schumacher et al., 2003).

During the hand-selection phase, we also observed increased activity in key nodes of a canonical network termed the "salience network" (Seeley et al., 2007; Sridharan et al., 2008; Habas et al., 2009), namely in the anterior cingulate (RCZa) and orbital fronto-insular cortex, especially in lOFC. By virtue of receiving input from all modalities of high-order sensory cortices as well as from limbic structures, this network has a leading role in predicting and evaluating the costs and benefits associated with an action based on interoceptive and exteroceptive sensory information and its anticipated utility (McCoy and Platt, 2005; O'Doherty, 2007; Rushworth et al., 2007; Kable and Glimcher 2009). Accordingly, lOFC would have contributed primarily to evaluation of the benefits of the sensory consequences of the manual actions during the hand-selection phase driven by the competing action representations, whereas the RCZa would have evaluated the costs of implementing each of those, including conflict issues (Ridderinkhof et al., 2004a). However, to support a decision about which action representation to favor, such utility evaluation must eventually end in a switching from exploration to exploitation of the outcome of the evaluation. Recent evidence suggests that the anterior cingulate cortex can produce signals specific of the first distinct reward, which marks the end of exploration (Quilodran et al., 2008). By directly and indirectly providing directions for the operational states of dlPFC as well as for the dorsal premotor areas (Miyachi et al., 2005; Tanji and Hoshi, 2008), it is likely that such signals supported the implementation of the desired action representation in the bimanual trials. Indeed, evidence in macaques during reaching indicates that arm selection involves neural processes in the dorsal premotor and supplementary motor areas (Hoshi and Tanji, 2000, 2004).

\section{Model for hand selection in the bimanual trials}

Under the supposition that optional action representations were simultaneously implemented initially in the target-chasing task and that a left lateralized parietal-premotor network governs the task regardless of the prime actor, the implementation of the right hand as a prime actor would depend on a withdrawal of commands from this network to the right SMC. We propose that this is implemented by prefrontal signals suppressing processes in the SMA that functionally links the right and left dorsolateral premotor cortex. This would impede the information flow between the left-lateralized parietal-premotor network and the right dorsolateral premotor areas that otherwise would drive the right SMC. The SMA (and the CCZ) has indeed a central position for controlling the routing of information both between hemispheres (Liu et al., 2002) and between premotor areas and the SMC within the hemisphere (Dum and Strick, 2005). Conversely, the implementation of the left hand as the prime actor would depend on prefrontal signals primarily suppressing processes in the left CCZ while saving the transcallosal flow controlled by SMA cortex supporting left-hand dominance. The suppression of the left CCZ would impede information flow between the left SMC and the left parietal-premotor network governing the task and thereby withdraw commands to the left SMC. Indeed, the SMA shows stronger BOLD activity when the left hand primarily acts than when the right does, whereas the CCZ is less active when the left hand functions as the prime actor than when the right does (Theorin and Johansson, 2007). Recent functional connectivity studies in right-handed humans based on transcranial magnetic brain stimulation further support the proposed asymmetric functional relationship between dorsolateral premotor areas of the left and right hemisphere and the contralateral SMC (Koch et al., 2006).

\section{Task set switching}

That RCZp and FIC together with ventral striatopallidum and putamen were activated during the switch from the hold task to the target-chasing task corroborates previous findings indicating that prefrontal brain areas can be transiently engaged around the time when the brain switches between tasks sets (Rushworth et al., 2002; Dosenbach et al., 2006; Sridharan et al., 2008). The RCZp and FIC have been identified previously as constituting core parts of a task set system in the brain (Dosenbach et al., 2006). The identified areas would likewise belong to components of the salience network considered responsible for switching between activation and deactivation of large-scale brain networks so as to support different general functional states of the brain (Sridharan et al., 2008). The involvement of the putamen and the ventral striatopallidum corroborates the view that basal ganglia and frontostriatal circuits are of importance for switching between task sets rather than resolving control issues within task sets or particular movements (Cools et al., 1984; Mink, 1996; Redgrave et al., 1999; Rushworth et al., 2002; Helmich et al., 2009).

The overall posterior localization of the prefrontal areas associated with task switching compared with hand selection indicates involvement of different neural substrates. Furthermore, the similar activity during the bimanual and unimanual trials in these areas indicates that the neural processes responsible for the task switching operated at a superordinate level with reference to processes responsible for the hand selection. That the areas associated with initiation of target chasing runs were less activated later during the runs suggest that the processes behind task switching operated at a superordinate level also with regard to the sensorimotor computations responsible for initiation and execution of the sequential actions during ongoing target chasing. Presumably, the dorsal parietal-premotor network, active throughout the runs, principally handled these computations, including the parametric specification of the motor commands to the current target positions (Kawashima et al., 1995; Grafton et al., 1996; Wise et al., 1997; Burnod et al., 1999). 


\section{References}

Bunge SA (2004) How we use rules to select actions: a review of evidence from cognitive neuroscience. Cogn Affect Behav Neurosci 4:564-579.

Burnod Y, Baraduc P, Battaglia-Mayer A, Guigon E, Koechlin E, Ferraina S, Lacquaniti F, Caminiti R (1999) Parieto-frontal coding of reaching: an integrated framework. Exp Brain Res 129:325-346.

Cao J (1999) The size of the connected components of excursion sets of $\chi^{2}$, $\mathrm{t}$ and F fields. Adv Appl Probab 31:579-595.

Cisek P (2007) Cortical mechanisms of action selection: the affordance competition hypothesis. Philos Trans R Soc Lond B Biol Sci 362:1585-1599.

Cisek P, Kalaska JF (2005) Neural correlates of reaching decisions in dorsal premotor cortex: specification of multiple direction choices and final selection of action. Neuron 45:801-814.

Cools AR, van den Bercken JH, Horstink MW, van Spaendonck KP, Berger HJ (1984) Cognitive and motor shifting aptitude disorder in Parkinson's disease. J Neurol Neurosurg Psychiatry 47:443-453.

Dosenbach NU, Visscher KM, Palmer ED, Miezin FM, Wenger KK, Kang HC, Burgund ED, Grimes AL, Schlaggar BL, Petersen SE (2006) A core system for the implementation of task sets. Neuron 50:799-812.

Dum RP, Strick PL (2005) Frontal lobe inputs to the digit representations of the motor areas on the lateral surface of the hemisphere. J Neurosci 25:1375-1386.

Dutta A, Proctor RW (1992) Persistence of stimulus-response compatibility effects with extended practice. J Exp Psychol Learn Mem Cogn 18:801-809.

Fincham JM, Carter CS, van Veen V, Stenger VA, Anderson JR (2002) Neural mechanisms of planning: a computational analysis using event-related fMRI. Proc Natl Acad Sci U S A 99:3346-3351.

Fitts PM, Deininger RL (1954) S-R compatibility: correspondence among paired elements within stimulus and response codes. J Exp Psychol 48:483-492.

Forman SD, Cohen JD, Fitzgerald M, Eddy WF, Mintun MA, Noll DC (1995) Improved assessment of significant activation in functional magnetic resonance imaging (fMRI): use of a cluster-size threshold. Magn Reson Med 33:636-647.

Friston KJ, Holmes AP, Price CJ, Büchel C, Worsley KJ (1999) Multisubject fMRI studies and conjunction analyses. Neuroimage 10:385-396.

Geschwind N (1975) The apraxias: neural mechanisms of disorders of learned movement. Am Scientist 63:188-195.

Goel V, Tierney M, Sheesley L, Bartolo A, Vartanian O, Grafman J (2007) Hemispheric specialization in human prefrontal cortex for resolving certain and uncertain inferences. Cereb Cortex 17:2245-2250.

Gold JI, Shadlen MN (2001) Neural computations that underlie decisions about sensory stimuli. Trends Cogn Sci 5:10-16.

Goldberg E, Podell K, Lovell M (1994) Lateralization of frontal lobe functions and cognitive novelty. J Neuropsychiatry Clin Neurosci 6:371-378.

Grafton ST, Fagg AH, Woods RP, Arbib MA (1996) Functional anatomy of pointing and grasping in humans. Cereb Cortex 6:226-237.

Guiard Y (1987) Asymmetric division of labor in human skilled bimanual action: the kinematic chain as a model. J Mot Behav 19:486-517.

Habas C, Kamdar N, Nguyen D, Prater K, Beckmann CF, Menon V, Greicius MD (2009) Distinct cerebellar contributions to intrinsic connectivity networks. J Neurosci 29:8586-8594.

Hadland KA, Rushworth MF, Passingham RE, Jahanshahi M, Rothwell JC (2001) Interference with performance of a response selection task that has no working memory component: an rTMS comparison of the dorsolateral prefrontal and medial frontal cortex. J Cogn Neurosci 13:1097-1108.

Helmich RC, Aarts E, de Lange FP, Bloem BR, Toni I (2009) Increased dependence of action selection on recent motor history in Parkinson's disease. J Neurosci 29:6105-6113.

Hoshi E, Tanji J (2000) Integration of target and body-part information in the premotor cortex when planning action. Nature 408:466-470.

Hoshi E, Tanji J (2004) Differential roles of neuronal activity in the supplementary and presupplementary motor areas: from information retrieval to motor planning and execution. J Neurophysiol 92:3482-3499.

Iacoboni M, Woods RP, Mazziotta JC (1996) Brain-behavior relationships: evidence from practice effects in spatial stimulus-response compatibility. J Neurophysiol 76:321-331.

Jiang Y, Kanwisher N (2003) Common neural substrates for response selection across modalities and mapping paradigms. J Cogn Neurosci 15:1080-1094.
Johansson RS, Theorin A, Westling G, Andersson M, Ohki Y, Nyberg L (2006) How a lateralized brain supports symmetrical bimanual tasks. PLoS Biol 4:e158.

Kable JW, Glimcher PW (2009) The neurobiology of decision: consensus and controversy. Neuron 63:733-745.

Kawashima R, Roland PE, O’Sullivan BT (1995) Functional anatomy of reaching and visuomotor learning: a positron emission tomography study. Cereb Cortex 5:111-122.

Koch G, Franca M, Del Olmo MF, Cheeran B, Milton R, Alvarez Sauco M, Rothwell JC (2006) Time course of functional connectivity between dorsal premotor and contralateral motor cortex during movement selection. J Neurosci 26:7452-7459.

Kornblum S, Hasbroucq T, Osman A (1990) Dimensional overlap: cognitive basis for stimulus-response compatibility: a model and taxonomy. Psychol Rev 97:253-270.

Leiguarda RC, Marsden CD (2000) Limb apraxias: higher-order disorders of sensorimotor integration. Brain 123:860-879.

Liu J, Morel A, Wannier T, Rouiller EM (2002) Origins of callosal projections to the supplementary motor area (SMA): a direct comparison between pre-SMA and SMA-proper in macaque monkeys. J Comp Neurol 443:71-85.

MacNeilage PF (1987) The evolution of hemispheric specialization for manual function and language. In: Higher brain functions (Wise SP, ed), pp 285-309. New York: Wiley.

Mazurek ME, Roitman JD, Ditterich J, Shadlen MN (2003) A role for neural integrators in perceptual decision making. Cereb Cortex 13:1257-1269.

McCoy AN, Platt ML (2005) Expectations and outcomes: decision-making in the primate brain. J Comp Physiol A Neuroethol Sens Neural Behav Physiol 191:201-211.

Miller EK, Cohen JD (2001) An integrative theory of prefrontal cortex function. Annu Rev Neurosci 24:167-202.

Mink JW (1996) The basal ganglia: focused selection and inhibition of competing motor programs. Prog Neurobiol 50:381-425.

Miyachi S, Lu X, Inoue S, Iwasaki T, Koike S, Nambu A, Takada M (2005) Organization of multisynaptic inputs from prefrontal cortex to primary motor cortex as revealed by retrograde transneuronal transport of rabies virus. J Neurosci 25:2547-2556.

O’Doherty JP (2007) Lights, camembert, action! The role of human orbitofrontal cortex in encoding stimuli, rewards, and choices. Ann N Y Acad Sci 1121:254-272.

Oldfield RC (1971) The assessment and analysis of handedness: the Edinburgh inventory. Neuropsychologia 9:97-113.

Picard N, Strick PL (2001) Imaging the premotor areas. Curr Opin Neurobiol 11:663-672.

Quilodran R, Rothé M, Procyk E (2008) Behavioral shifts and action valuation in the anterior cingulate cortex. Neuron 57:314-325.

Redgrave P, Prescott TJ, Gurney K (1999) The basal ganglia: a vertebrate solution to the selection problem? Neuroscience 89:1009-1023.

Richards L, Chiarello C (1997) Activation without selection: parallel right hemisphere roles in language and intentional movement? Brain Lang 57:151-178.

Ridderinkhof KR, Ullsperger M, Crone EA, Nieuwenhuis S (2004a) The role of the medial frontal cortex in cognitive control. Science 306:443-447.

Ridderinkhof KR, van den Wildenberg WP, Segalowitz SJ, Carter CS (2004b) Neurocognitive mechanisms of cognitive control: the role of prefrontal cortex in action selection, response inhibition, performance monitoring, and reward-based learning. Brain Cogn 56:129-140.

Rowe JB, Toni I, Josephs O, Frackowiak RS, Passingham RE (2000) The prefrontal cortex: response selection or maintenance within working memory? Science 288:1656-1660.

Rushworth MF, Hadland KA, Paus T, Sipila PK (2002) Role of the human medial frontal cortex in task switching: a combined fMRI and TMS study. J Neurophysiol 87:2577-2592.

Rushworth MF, Behrens TE, Rudebeck PH, Walton ME (2007) Contrasting roles for cingulate and orbitofrontal cortex in decisions and social behaviour. Trends Cogn Sci 11:168-176.

Sailer U, Flanagan JR, Johansson RS (2005) Eye-hand coordination during learning of a novel visuomotor task. J Neurosci 25:8833-8842.

Schall JD, Thompson KG (1999) Neural selection and control of visually guided eye movements. Annu Rev Neurosci 22:241-259.

Schluter ND, Krams M, Rushworth MF, Passingham RE (2001) Cerebral 
dominance for action in the human brain: the selection of actions. Neuropsychologia 39:105-113.

Schumacher EH, Elston PA, D'Esposito M (2003) Neural evidence for representation-specific response selection. J Cogn Neurosci 15:1111-1121.

Seeley WW, Menon V, Schatzberg AF, Keller J, Glover GH, Kenna H, Reiss AL, Greicius MD (2007) Dissociable intrinsic connectivity networks for salience processing and executive control. J Neurosci 27:2349-2356.

Sridharan D, Levitin DJ, Menon V (2008) A critical role for the right frontoinsular cortex in switching between central-executive and default-mode networks. Proc Natl Acad Sci U S A 105:12569-12574.

Talairach J, Tournoux P (1988) Co-planar stereotaxic atlas of the human brain. Three-dimensional proportional system: an approach to cerebral imaging. New York: Thieme.

Tanji J, Hoshi E (2008) Role of the lateral prefrontal cortex in executive behavioral control. Physiol Rev 88:37-57.

Theorin A, Johansson RS (2007) Zones of bimanual and unimanual preference within human primary sensorimotor cortex during object manipulation. Neuroimage 36 [Suppl 2]:T2-T15.
Tzourio-Mazoyer N, Landeau B, Papathanassiou D, Crivello F, Etard O, Delcroix N, Mazoyer B, Joliot M (2002) Automated anatomical labeling of activations in SPM using a macroscopic anatomical parcellation of the MNI MRI single-subject brain. Neuroimage 15:273-289.

Weiss PH, Rahbari NN, Lux S, Pietrzyk U, Noth J, Fink GR (2006) Processing the spatial configuration of complex actions involves right posterior parietal cortex: an fMRI study with clinical implications. Hum Brain Mapp 27:1004-1014.

Wise SP, Murray EA (2000) Arbitrary associations between antecedents and actions. Trends Neurosci 23:271-276.

Wise SP, Boussaoud D, Johnson PB, Caminiti R (1997) Premotor and parietal cortex: corticocortical connectivity and combinatorial computations. Annu Rev Neurosci 20:25-42.

Worsley KJ, Andermann M, Koulis T, MacDonald D, Evans AC (1999) Detecting changes in nonisotropic images. Hum Brain Mapp 8:98-101.

Worsley KJ, Liao CH, Aston J, Petre V, Duncan GH, Morales F, Evans AC (2002) A general statistical analysis for fMRI data. Neuroimage $15: 1-15$. 